\title{
Shifts in microbiota species and fermentation products in a dietary model enriched in fat and sucrose
}

Etxeberria $\mathrm{U}^{1}$, Arias $\mathrm{N}^{2}$, Boqué $\mathrm{N}^{3}$, Macarulla $\mathrm{MT}^{2,4}$, Portillo $\mathrm{MP}^{2,4}$, Milagro $\mathrm{FI}^{1,4}$, Martinez JA ${ }^{1,4, *}$

${ }^{1}$ Department of Nutrition, Food Science and Physiology, University of Navarra, Pamplona, Spain

${ }^{2}$ Nutrition and Obesity group, Department of Nutrition and Food Sciences, Faculty of Pharmacy, University of the Basque Country (UPV/EHU), Paseo de la Universidad 7, 01006 Vitoria, Spain

${ }^{3}$ Centro Tecnológico de Nutrición y Salud (CTNS), Av. Universitat 1, 43204 Reus, Spain

${ }^{4}$ CIBERobn Fisiopatología de la Obesidad y Nutrición (CIBERobn), Instituto de Salud Carlos III, 28029 Madrid, Spain

*Professor J.Alfredo Martínez

Department of Nutrition, Food Science and Physiology, University of Navarra, C/Irunlarrea, s/n, 31008, Pamplona, Navarra (Spain); Email address: jalfmtz@unav.es

\begin{abstract}
The gastrointestinal tract harbours a "superorganism" called the gut microbiota, which is known to play a crucial role in the onset and development of diverse diseases. This internal ecosystem, far from being a static environment, could be willingly manipulated by diet and dietary components. Feeding animals with high-fat sucrose diets entails diet-induced obesity, a model which is usually used in research to mimic the obese phenotype of Western societies.
\end{abstract}

The aim of the present study was to identify gut microbiota dysbiosis and associated metabolic changes produced in 5 male Wistar rats fed a high-fat sucrose (HFS) diet for six weeks and to compare it with the basal microbial composition. For this purpose, DNA extracted from faeces at baseline and after the treatment was analysed by amplification of the V4-V6 region of the 16S ribosomal DNA (rDNA) gene using 454 pyrosequencing. Short-chain fatty acids (SCFA), acetate, propionate and butyrate, were also evaluated by gas chromatography-mass spectrometry (GC-MS).

At the end of the treatment, gut microbiota composition significantly differed at phylum level (Firmicutes, Bacteroidetes and Proteobacteria) and class level (Erisypelotrichi, Deltaproteobacteria, Bacteroidia and Bacilli). Interestingly, Clostridia class showed a significant decrease after the HFS-diet treatment, which correlated with visceral adipose tissue, and is likely mediated by dietary carbohydrates. Of particular interest, Clostridium cluster XIVa species were significantly reduced and changes were identified in the relative abundance of other specific bacterial species (Mitsuokella jalaludinii, Eubacterium ventriosum, Clostridium sp. FCB90-3, Prevotella nanceiensis, Clostridium fusiformis, Clostridium sp. BNL1100 and Eubacterium cylindroides) that, in some cases, showed opposite trends to their relative families.

These results highlight the relevance of characterizing gut microbial population differences at species level and contribute to understand the plausible link between the 
diet and specific gut bacterial species that are able to influence the inflammatory status, intestinal barrier function and obesity development.

Keywords: gut microbiota, pyrosequencing, high-fat sucrose diet, short chain fatty acids, Erysipelotrichi

\section{Abbreviations}

HFS, high-fat sucrose;16S rDNA, 16S ribosomal DNA; SCFA, short-chain fatty acids; GC-MS, gas chromatography-mass spectrometry; C, standard-chow diet; PCI, positive chemical ionization; PCA, principal component analysis; SD, standard diet; HF, highfat diet; WD, western diet.

Running head: Gut microbial species and fermentation products modification after a high-fat sucrose diet 


\section{Introduction}

Obesity and associated clinical complications are a global health threat (Azagury and Lautz, 2011). The prevalence of obesity epidemics has a multifactorial origin that results from the interaction between genetics and environmental factors (Martinez et al., 2012). Recently, gut microbiota has attracted growing interest as a new contributory factor to obesity (Tagliabue and Elli, 2013). The adult intestine is home for an approximately 100 trillion microbes, with hundreds or thousands of species, occurring in the colonic region, whose density is estimated to be at $10^{11}$ to $10^{12}$ per ml (Million et al., 2013). This gut microbial population has been implicated in a range of essential host physiological processes (Evans et al., 2013; Tremaroli and Backhed, 2012). Moreover, due to the straight interactions between host and microbes, the gut microbiota dysbiosis has been associated to a number of diseases such as, diabetes, non-alcoholic fatty liver disease and atherosclerosis (Fukuda and Ohno, 2013; Y Lee, 2013). In this sense, studies directed to investigate the complex association between composition and functionality of gut microbes with obesity are emerging (Cani and Delzenne, 2011; Ley, 2010). Consumption of high-caloric diets is one of the driving forces for obesity development, producing not only an energy imbalance, but also a drastic impact on the microbial community residing the gastrointestinal tract (Duca et al., 2013; Wu et al., 2011). Despite the fact that the surrounding physiological mechanisms are not fully understood, high energy yielding diets rich in fat and sugars have been closely related to obesity (Lomba et al., 2010). In this context, further investigation of the impact of these types of diets on gut microbiota and their metabolic consequences is required (Daniel et al., 2013; Duca et al., 2013), in order to identify changes caused in the gut that could be involved in the development of the disease. Indeed, the relevance of the intestinal bacteria in metabolism is not only due to composition per se, but also to the metabolic activities performed by them. Thus, SCFA including acetate, propionate and butyrate, are microbial-derived fermentation products that are primarily generated in the large intestine from the metabolism of non-digestible polysaccharides, including resistant starch, non-starch polysaccharides and oligosaccharides (Flint et al., 2008). Furthermore, these microbial-derived products are believed to positively regulate several host metabolic pathways (den Besten et al., 2013; Layden et al., 2013). Predominant bacterial groups described as major producers of butyrate are Firmicutes, whereas propionate and acetate are mainly produced by Bacteroidetes (Macfarlane and Macfarlane, 2003). A number of studies have previously reported an increase in faecal SCFA concentrations in obese people compared to lean subjects (Ley et al., 2005; Ley et al., 2006; Turnbaugh et al., 2006). The changes in the proportion of the different bacterial species have been hypothesized to modify the efficiency of energy extraction from the diet (den Besten et al., 2013), resulting in a more pro-inflammatory luminal environment that could contribute to the progression of low chronic inflammation and metabolic disorders (Cani et al., 2013).

Accordingly, as a consequence of the interplay among diet, gut microbiota and host metabolism, this study was focused on characterising microbial community modifications and metabolic alterations in rats fed an obesogenic diet based on a highfat sucrose intake. GC-MS approach was used to detect SCFA production levels in faeces, while gut microbiota profiling was carried out by the high-throughput $16 \mathrm{~S}$ rDNA amplicon sequencing. 


\section{Material and methods}

\section{Animals and diets}

The experiment was conducted on five male Wistar rats supplied from Harlan Ibérica (Barcelona, Spain), with an average initial body weight of $194 \pm 2 \mathrm{~g}$ and was performed in accordance with the Ethical Committee of the University of the Basque Country (document reference CUEID CEBA/30/2010), following the European regulations (European Convention-Strasburg 1986, Directive 2003/65/EC and Recommendation 2007/526/EC). Animals were single-housed in polycarbonate cages (Techniplast Gazzada, Guguggiate, Italy) and kept in a temperature-controlled $\left(22 \pm 2{ }^{\circ} \mathrm{C}\right)$ isolated room under a 12:12-h artificial light/dark cycle (light on at 21:00). Rats were fed a standard-chow diet $(\mathrm{C} ; 2.9 \mathrm{kcal} / \mathrm{g}$ ) from Harlan Ibérica (ref. 2014, Barcelona, Spain) during an adaptation period of four days and then changed to a high-fat sucrose obesogenic diet (HFS; $4.6 \mathrm{kcal} / \mathrm{g}$ ) purchased from Harlan Ibérica (ref. TD. 06415) for 6 weeks. The $\mathrm{C}$ diet provided $20 \%$ of energy as proteins, $67 \%$ as carbohydrates and $13 \%$ as fat by dry weight, whereas the HFS diet contained $19 \%$ proteins, $36 \%$ carbohydrates and $45 \%$ as fat (Table 1). All animals had free access to food and water. Body weight and food intake were measured daily.

Table 1. Composition of the diets: control diet (2014, Harlan Teklad Global 14\% Protein Rodent Maintenance Diet), High-fat sucrose diet (TD. 06415, Teklad Research purified diet).

\begin{tabular}{lcc}
\hline & $\begin{array}{c}\text { Control diet } \\
(\mathbf{2 0 1 4})\end{array}$ & $\begin{array}{c}\text { High-fat sucrose diet } \\
\text { (TD. 06415) }\end{array}$ \\
\hline Energy (Kcal/g) & 2.9 & 4.6 \\
Protein (Energy \%) & 20 & 19 \\
Carbohydrate (Energy \%) & 67 & 36 \\
Starch* (g/kg) & 480 & 85 \\
Sucrose** (g/kg) & Traces & 200 \\
Fat (Energy \%) & 13 & 45 \\
Saturated $(\%)$ & 18 & 36 \\
Monounsaturated $(\%)$ & 20 & 47 \\
Polyunsaturated $(\%)$ & 62 & 17 \\
Fiber content*** (g/kg) & 221 & 58 \\
\hline & & \\
* Unrefined starch from wheat and corn in 2014; refined corn starch in TD.06415 \\
** No added sucrose in 2014; trace amounts of simple sugars from grain ingredients \\
** Calculated neutral detergent fiber in 2014 \& cellulose in TD.06415
\end{tabular}

\section{Faeces collection}

Fresh faecal samples were collected at fourth day of the adaptation period and at the end of the intervention period, early in the morning, by abdominal massage and prior to the overnight fasting. Samples were collected in $15 \mathrm{ml}$ Falcon tubes and frozen at $-80{ }^{\circ} \mathrm{C}$ for future analyses.

Tissue collection 
At the end of the experimental period ( 6 weeks), animals were fasted overnight and sacrificed under anaesthesia (chloral hydrate) by cardiac exsanguination between 9:00 and 12:00 a.m. White adipose tissue from different anatomical locations (epididymal, perirenal, mesenteric and subcutaneous) was collected, weighted and then stored frozen at $-80^{\circ} \mathrm{C}$.

\section{Serum biochemical measurements}

Glucose was measured using a Glucose oxidase/peroxidase kit (Ref. 110504, BioSystems, Barcelona, Spain), insulin levels were determined by using specific ELISA kit according to the protocol described by the manufacturer (Ref. 10-1250-01, Mercodia AB, Spain).

\section{Gut microbiota analysis}

\section{DNA extractions}

Total DNA from stool subsamples (180-220 mg) was extracted using a QIAmp DNA Stool Mini Kit (Qiagen, Hilden, Germany), following supplier`s instructions. Briefly, the method consists on the lysis of the bacterial cells within the faecal material in ASL buffer, which is specifically developed to remove inhibitory substances from stool samples, adsorption of impurities to InhibitEX reagent and purification of the DNA on a spin column. Eluted DNA was quantified using a NanoDrop ND-1000 spectrophotometer (NanoDrop Technologies) and stored at $-20^{\circ} \mathrm{C}$ until analysis.

\section{High-throughput amplicon pyrosequencing}

Extracted DNA was used to amplify and to sequence pooled amplicons specific for the $\sim 560 \mathrm{bp}$ of the V4-V6 region of the bacterial 16S rDNA gene, which were generated using 16S-0515F (5'-TGYCAGCMGCCGCGGTA-3') and 16S-1061R (5'-TCACGRCACGAGCTGACG-3') primers tailed on each end with the Roche multiplex identifiers. This barcode-based primer approach allowed sequencing of multiple samples in a single sequencing run without the need for physical partitioning. Specific PCR conditions for the pfu DNA polymerase (Ref. M774, Promega, Madison, WI) were performed in order to get the amplicons following manufacturer's instructions. PCR products were then cleaned using the PCR purification Kit (Qiagen) and DNA concentrations were determined using the Quant-iT PicoGreen dsDNA reagent and kit (Invitrogen, California, USA) as previously described (Milagro et al., 2011). The final pool of cleaned amplicons combined in equimolar ratios into a single tube was prepared taking $100 \mathrm{ng}$ from each sample in a total volume of $55.61 \mu \mathrm{l}$. Single- ended pyrosequencing was carried out by Beckman Coulter Genomics (Danvers, MA, USA) on a 454 Life Sciences Genome Sequencer FLX instrument (Roche), following one pippin prep, for size selection of the amplicons of interest. The sequence of the 10 amplicon libraries produced 16S rDNA reads ranging from 11, 808 to 34,934 , generating read lengths with an average of $517 \mathrm{bp}$. The reads were clustered using the open source MIRA (Chevreux et al., 2004) assembler in EST mode with an identity threshold of 0.98 , minimum coverage of $1 \mathrm{x}$ and minimal contig length of $400 \mathrm{bp}$, contigs and singletons were blasted against a curated copy of the RDP database and blast results were provided to MEGAN (Huson et al., 2011) for 
taxonomic classification. Construction of libraries and sequencing were all provided as a custom service of Beckman Coulter Genomics.

Short chain fatty acids analysis

\section{Reagents and standards}

Diethyl ether CHROMASOLV for HPLC was from Sigma-Aldrich (Steinheim, Germany), while water was produced in a Milli-Q purification system (Millipore, Molsheim, France). Phosphoric acid was purchased from Sigma-Aldrich. Analytical standards sodium acetate, sodium propionate, sodium butyrate and propionic acid- $\mathrm{d}_{6}$ (98 atom \%D) was from Sigma-Aldrich.

\section{Calibration}

Individual standard stock solutions of the SCFA of $2000 \mathrm{mg} / \mathrm{L}$ were prepared in water and stored at $-20^{\circ} \mathrm{C}$ until use. Immediately before assay, dilutions in acid water $(0.5 \%$ phosphoric acid) were performed to generate a calibration series at concentration range of 0.1 to $500 \mathrm{mg} / \mathrm{L}$ of the three standards. Internal standard (propionic acid- $\mathrm{d}_{6}$, 98 atom \%D) was prepared at a concentration of $2 \mathrm{mg} / \mathrm{L}$ in acid water. A volume of $500 \mu \mathrm{L}$ of this solution was added to calibrators before extraction.

\section{Sample preparations}

Exactly $500 \mu \mathrm{L}$ of acid water (containing internal standard) was added to $25 \mathrm{mg}$ of each baseline and final lyophilized sample. Overnight lyophilization was carried out protecting samples from light. Mixture was shaken for 1 minute. Afterwards, extraction was carried out with $500 \mu \mathrm{L}$ of diethyl ether followed by 1 minute of shaking and centrifugation at $4^{\circ} \mathrm{C}$ at $14000 \mathrm{rpm}$ for $10 \mathrm{~min}$. Supernatants were analysed.

\section{Gas chromatography-mass spectrometry analysis}

Samples were analysed in a 7890A Series gas chromatograph coupled to a 7000 GC/MS Triple Quad (Agilent Technologies, Santa Clara, U.S.A.). Chromatographic column was a J\&W Scientific HP-FFAP ( $30 \mathrm{~m}$ x $0.25 \mathrm{~mm}$ i.d., $0.25 \mu \mathrm{m}$ film) (Agilent Technologies). A volume of $1 \mu 1$ of sample was automatically injected into a split/splitless inlet (in splitless mode), which was kept at $175^{\circ} \mathrm{C}$. Helium $(99.999 \%$ purity) was used as a carrier gas, at a flow rate of $1 \mathrm{ml} / \mathrm{min}$ in constant flow mode. The oven program was set at an initial temperature of $90^{\circ} \mathrm{C}$, increased to $150^{\circ} \mathrm{C}$ at a rate of $12^{\circ} \mathrm{C} / \mathrm{min}$, then increased to $240^{\circ} \mathrm{C}$ at a rate of $20^{\circ} \mathrm{C} / \mathrm{min}$, and held at $240^{\circ} \mathrm{C}$ for $5 \mathrm{~min}$. Ionization was done by positive chemical ionization (PCI) with methane gas. SIM mode was used for the acquisition. GC-QqQ MassHunter acquisition software (Agilent Technologies) was used for data acquisition.

\section{Statistical analysis}

All results are expressed as the mean \pm standard error of the mean. Statistical significance between groups was assessed by Paired-Samples T-Test. Benjamini- 
Hochberg multiple testing corrections was applied for variables presenting probabilities of $p<0.05$, unadjusted $p$ values which were lower than the calculated critical values were considered statistically significant. SPSS 15.0 software (Chicago, USA) was used to perform statistical analysis and principal component analysis (PCA) was performed using XLSTAT software (http://www.xlstat.com).

\section{Results}

\section{Effect of a high-fat sucrose diet on weight and bacterial community structure}

After six weeks of HFS diet, rats gained an average weight of $176 \pm 7 \mathrm{~g}$. At the end of the intervention, animals presented significantly higher final body weight $(p<0.05)$ when compared to baseline (Figure 1). A total of 209, 709 reads (84, 604 at baseline and 125,105 at the end of the treatment) were obtained when V4-V6 regions of $16 \mathrm{~S}$ rDNA gene were sequenced to assess the impact of the HFS diet on bacterial community. Across all samples measured at the beginning and at the end of the experiment, the average length of quality sequences ranged from 499 to $532 \mathrm{bp}$. In faeces of the experimental rats, members from Firmicutes phyla were predominant, representing from $61-96 \%$ of $16 \mathrm{~S}$ rDNA gene sequences, followed by Bacteroidetes, the second most abundant phyla (2-37\%). These two divisions were more than $90 \%$ of the sequences before and after the treatment period.

There were no differences in total bacterial 16S rDNA frequencies of the samples between baseline and the end of the treatment. However, statistically significant increases were found for Firmicutes $(35 \%)$ and Proteobacteria $(509 \%)$ phyla at the end of the experimental period. Accordingly, Bacteroidetes were reduced on average by $69 \%$. Relative abundance of other phyla found in lower levels, such as Actinobacteria (from $0.7 \%$ to $0.4 \%$ ), Clamydiae/Verrucomicrobia group (from $0.2 \%$ to $1 \%$ ), Tenericutes (from $0.4 \%$ to $2.7 \%$ ), Cyanobacteria (from $0.3 \%$ to $0.4 \%$ ), Deferribacteres $(0.5 \%$ ) and unclassified bacteria (from $0.4 \%$ to $0.3 \%$ ), were also statistically different between baseline and the end of the HFS diet intervention, but changes were non-significant. Faecal bacterial populations identified before and after the HFS-diet treatment at phylum level are illustrated in Figure $\mathbf{2 a}$ and $\mathbf{2} \mathbf{b}$.

At class level, significant differences in major bacterial groups were detected when comparing gut microbiota composition at the beginning and at the end of the intervention. A "bloom" in Erysipelotrichi, one of the major classes within the Firmicutes phylum, was observed and a significant increase of Deltaproteobacteria, a class of Proteobacteria was identified. Although no statistically significant outcomes after adjusting by multiple comparison test were found, an enhancement of Gammaproteobacteria (433\%) was also identified (Table 2). Surprisingly, the levels of Clostridia (-30\%) and Bacilli (-79\%) classes, belonging both to the phylum Firmicutes, were decreased. Unexpectedly, a positive correlation was detected between Clostridia levels and animals' visceral adipose tissue percentage $(p=0.037$; $\mathrm{r}=0.900$ ) (Figure 3). On the other hand, a significant fall in the relative abundance of Bacteroidia (-69\%), a member of Bacteroidetes phyla, was found after the intervention period (Table 2). 
Table 2. Taxa frequencies and percentage of change (\%) of the relative abundance of bacterial groups at class level in rats at baseline and after a 6 week HFS diet treatment.

\begin{tabular}{|c|c|c|c|c|c|c|}
\hline Ribosomal Database Project classification & Bacterial group & HFS baseline & HFS after treatment & Change (\%) & $\begin{array}{c}\text { Unadjusted } \\
p \text { value }\end{array}$ & $\begin{array}{l}\text { Critical } \\
p \text { value }\end{array}$ \\
\hline Firmicutes; Clostridia & Clostridia & $0.572 \pm 0.015$ & $0.396 \pm 0.021$ & -30.7 & 0.001 & $*$ \\
\hline Bacteroidetes; Bacteroidetes; Bacteroidia & Bacteroidia & $0.350 \pm 0.011$ & $0.107 \pm 0.037$ & -69.4 & 0.001 & $*$ \\
\hline Firmicutes; Erysipelotrichi & Erysipelotrichi & $0.038 \pm 0.024$ & $0.425 \pm 0.064$ & 1025.4 & 0.002 & $*$ \\
\hline Firmicutes; Bacilli & Bacilli & $0.015 \pm 0.002$ & $0.003 \pm 0.001$ & -79.8 & 0.002 & $*$ \\
\hline Proteobacteria; delta/epsilon subdivisions; Deltaproteobacteria & Deltaproteobacteria & $0.002 \pm 0.001$ & $0.026 \pm 0.005$ & 1575.7 & 0.011 & $*$ \\
\hline Proteobacteria; Gammaproteobacteria & Gammaproteobacteria & $0.001 \pm 0.000$ & $0.004 \pm 0.001$ & 433.0 & 0.020 & ns \\
\hline
\end{tabular}

Taxonomic breakdown of Wistar rats intestinal bacterial V4-V6 regions obtained by pyrosequencing before and after HFS diet treatment. All results are expressed as the taxa frequencies mean \pm SEM and the average of bacterial relative abundance change (\%) between final and baseline. Statistical analyses were performed using Paired- Samples T-test. Variables presenting probabilities of $\mathrm{p} \quad 0.05$ were applied the Benjamini-Hochberg multiple testing correction. Unadjusted $p$ values lower than the calculated critical $p$ values were considered statistically significant $(*)$. HFS, high- fat sucrose; SEM, standard error of the mean. 
At family level, the relative proportion of most of the detected bacterial groups (Lachnospiraceae, Eubacteriaceae, Lactobacillaceae, Porphyromonadaceae, Rikenellaceae and Clostridiales Family XIII incertae sedis) was lower at the end of the HFS diet intervention when compared to the baseline, although these results remained not statistically significant after applying the correction with the multiple comparison test (Table 3). However, a significant reduction in the Prevotellaceae $(-96 \%)$ family, within the Bacteroidetes division $(p<0.001)$, was detected. Furthermore, the Prevotellaceae/Bacteroidaceae ratio was significantly lower ( $p<0.001$ ) in the diet-induced overweight rats (Figure 4). In contrast, Desulfovibrionaceae (966\%), unclassified Erysipelotrichiciae (6055\%), Peptococcaceae (130\%) and Xanthomonadaceae (227\%) increased considerably (although not statistically significantly) after the treatment period (Table 3). 
Table 3. Taxa frequencies and percentage of change (\%) of the relative abundance of bacterial groups at family level in rats at baseline and after a 6 week HFS diet treatment.

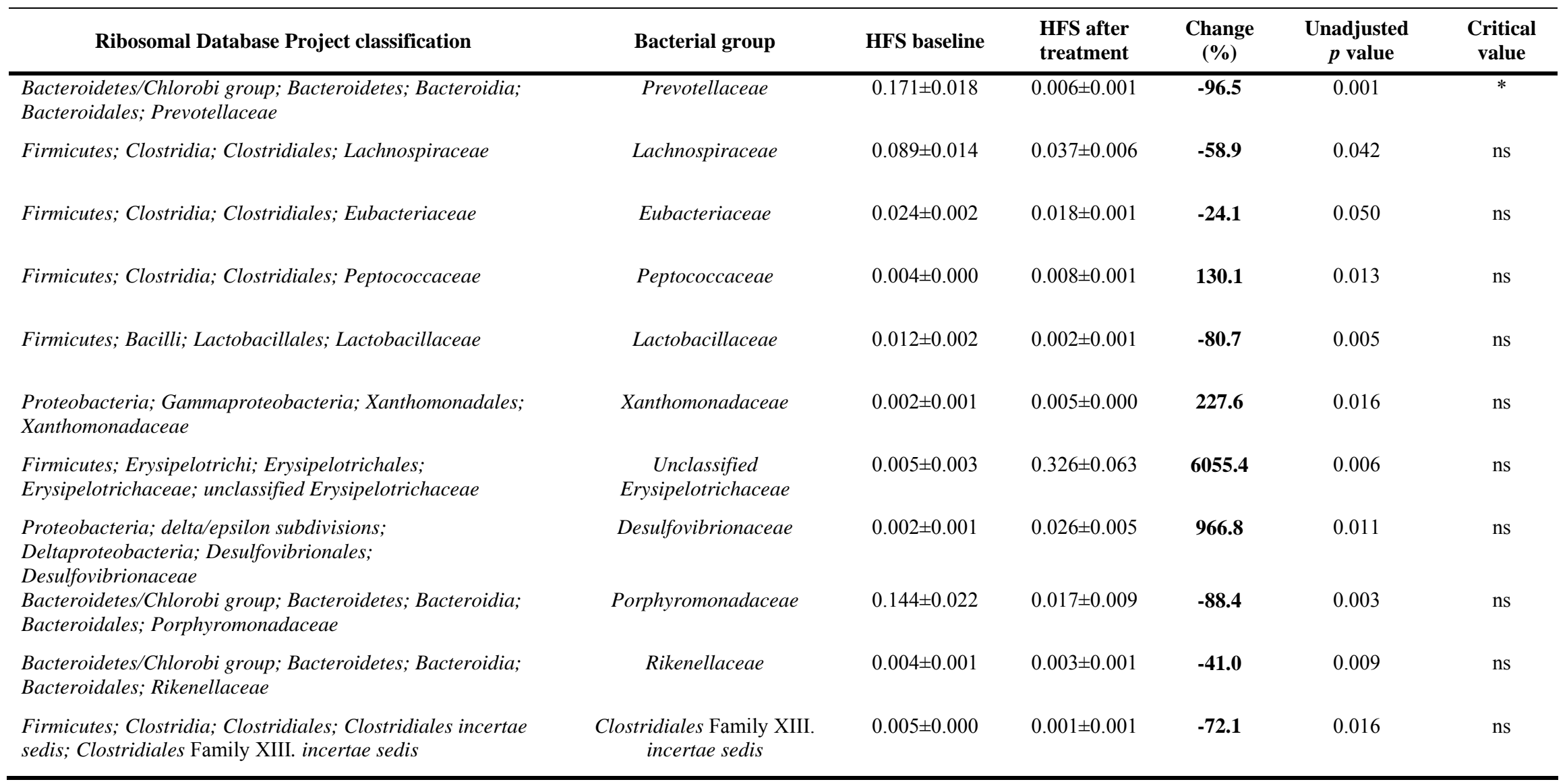

Taxonomic breakdown of Wistar rats intestinal bacterial V4-V6 regions obtained by pyrosequencing before and after HFS diet treatment. All results are expressed as the taxa frequencies mean \pm SEM and the average of bacterial relative abundance change (\%) between final and baseline. Statistical analyses were performed using Paired- Samples T-test. Variables presenting probabilities of $\mathrm{p} \quad 0.05$ were applied the Benjamini-Hochberg multiple testing correction. Unadjusted $p$ values lower than the calculated critical $p$ values were considered statistically significant $\left(^{*}\right)$. HFS, high-fat sucrose; SEM, standard error of the mean. 
A clustering of microbiota analysis by Principal Component Analysis (PCA) revealed that distribution of animals at baseline and after the treatment was characterized by differentiated bacterial families (Figure 5a and $\mathbf{5 b}$ ). Twenty six bacterial families represented faecal gut microbiota in control diet-fed lean rats, while twenty one groups of bacteria characterized gut microbial community in faces samples after the six week HFS diet intervention.

\section{Association of diet-induced overweight to signature of genera and species}

High-throughtput pyrosequencing enabled not only to detect a distinct microbial population pattern at higher division levels, but also made possible to identify significant alterations in the relative abundance of several bacterial genera and specific bacterial species in faeces of control diet-fed rats that were submitted to a high-fat sucrose diet treatment. Thus, Mitsuokella genus showed a statistically significant reduction concerning percentage of change (-90\%), and Maribacter (a genus within the Flavobacteriaceae family) presented a significant increase in its relative abundance $(455 \%)$ at the end of the treatment. In agreement with the aforementioned results, at species levels, relative abundance of Mitsuokella jalaludinii, was significantly lower in the HFS diet-induced overweight rats. Some bacterial species showed the opposite pattern to the families or genus they belong to. Thus, Eubacterium ventriosum, a bacterial species from the Eubacteriaceae family, was significantly increased (384\%), while the Eubacteriaceae family decreased (-24\%) in the HFS diet-fed rats. Similarly, Prevotella nanceiensis, from the Prevotellaceae family, was significantly enriched (322\%), and other Prevotella species also showed a considerable enhancement (Prevotella micans, 157\%; Prevotella marshii, 205\%) after 6 weeks of a HFS diet treatment. Moreover, although Clostridia class presented a reduction at the end of the experimental period, some species from this group were detected to be significantly enriched in overweight rats, such as Clostridum sp. FCB90-3 (414\%) and Clostridium sp. BNL1100 (219\%). In contrast, other Clostridium species showed significantly lower levels in the faeces of the diet-induced obese rats, such as Clostridium fusiformis $(-77 \%)$. Although the percentage of change was not statistically significant, when the relative abundance of bacteria was compared at baseline and after the HFS diet treatment, some bacterial species such as Eubacterium cylindroides (4072\%), Bilophila wadsworthia (406\%) and Clostridium algidixylanolyticum (235\%) substantially increased in the faeces of the diet-induced overweight rats, while other species showed a considerable decrease (Clostridium hathewayi, -85\%; Clostridium aerotolerans, $-68.9 \%$; Clostridium clariflavum, -89\%; Clostridium aminobutyricum, -74\%. Some members of the phylum Bacteroidetes were also found to extensively increase in the diet-induced obese rats (Bacteroides heparinolyticus, 204\%; Bacteroides sp. S-18, $1526 \%$; Bacteroides sp. dnLKV7, 427.8\%). The relative abundance of these microbes whose percentage changed in the faeces from the HFS diet-fed rats before and after the intervention is shown in Table 4. 
Table 4. Taxa frequencies and percentage of change (\%) of the relative abundance of bacterial groups at species level in rats at baseline and after a 6 week HFS diet treatment.

\begin{tabular}{|c|c|c|c|c|c|c|c|c|}
\hline Phyla & Family & Genus & Bacterial species & HFS baseline & $\begin{array}{l}\text { HFS after } \\
\text { treatment }\end{array}$ & Change (\%) & $\begin{array}{c}\text { Unadjusted } \\
p \text { value }\end{array}$ & $\begin{array}{c}\text { Critical } \\
\text { value }\end{array}$ \\
\hline Firmicutes & Veillonellaceae & Mitsuokella & Mitsuokella jalaludinii & $0.005 \pm 0.000$ & $0.000 \pm 0.000$ & -91.4 & 0.001 & * \\
\hline Firmicutes & Eubacteriaceae & Eubacterium & Eubacterium ventriosum & $0.001 \pm 0.000$ & $0.005 \pm 0.000$ & 384.2 & 0.001 & * \\
\hline Firmicutes & Clostridiaceae & Clostridium & Clostridium sp. FCB90-3 & $0.004 \pm 0.001$ & $0.005 \pm 0.000$ & 414.5 & 0.001 & * \\
\hline Bacteroidetes & Prevotellaceae & Prevotella & Prevotella nanceiensis & $0.001 \pm 0.000$ & $0.005 \pm 0.000$ & 322.3 & 0.001 & * \\
\hline Firmicutes & Clostridiaceae & Clostridia & Clostridium fusiformis & $0.005 \pm 0.000$ & $0.001 \pm 0.000$ & -77.1 & 0.001 & * \\
\hline Firmicutes & Clostridiaceae & Clostridium & Clostridium sp. BNL1100 & $0.002 \pm 0.000$ & $0.005 \pm 0.000$ & 219.8 & 0.001 & $*$ \\
\hline Firmicutes & Erysipelotrichaceae & Clostridium & Eubacterium cylindroides & $0.005 \pm 0.003$ & $0.191 \pm 0.041$ & 4072.1 & 0.009 & ns \\
\hline Firmicutes & Clostridiaceae & Clostridium & Clostridium hathewayi & $0.037 \pm 0.008$ & $0.005 \pm 0.001$ & -85.6 & 0.018 & ns \\
\hline Firmicutes & Clostridiaceae & Clostridium & Clostridium sp. C9 & $0.002 \pm 0.001$ & $0.004 \pm 0.001$ & 130.7 & 0.050 & ns \\
\hline Firmicutes & Clostridiaceae & Clostridium & Clostridium xylanovorans & $0.005 \pm 0.002$ & $0.002 \pm 0.001$ & -61.1 & 0.041 & ns \\
\hline Firmicutes & Clostridiaceae & Clostridium & Clostridium aerotolerans & $0.005 \pm 0.000$ & $0.002 \pm 0.001$ & -68.9 & 0.016 & ns \\
\hline Firmicutes & Clostridiaceae & Clostridium & Clostridium clariflavum & $0.004 \pm 0.001$ & $0.000 \pm 0.000$ & -89.5 & 0.017 & ns \\
\hline Firmicutes & Clostridiaceae & Clostridium & Clostridium aminobutyricum & $0.005 \pm 0.000$ & $0.001 \pm 0.001$ & -74.6 & 0.016 & ns \\
\hline Firmicutes & Clostridiaceae & Clostridium & Clostridium algidixylanolyticum & $0.001 \pm 0.001$ & $0.005 \pm 0.000$ & 235.4 & 0.017 & ns \\
\hline Firmicutes & $\begin{array}{l}\text { Clostridiales Family XIII. } \\
\text { Incertae Sedis }\end{array}$ & & [Eubacterium] infirmum & $0.005 \pm 0.000$ & $0.001 \pm 0.001$ & -72.8 & 0.016 & ns \\
\hline Firmicutes & Eubacteriaceae & Eubacterium & Eubacterium plexicaudatum & $0.008 \pm 0.001$ & $0.002 \pm 0.001$ & -69.7 & 0.001 & ns \\
\hline Firmicutes & Eubacteriaceae & Eubacterium & Eubacterium fissicatena & $0.005 \pm 0.000$ & $0.002 \pm 0.001$ & -69.0 & 0.016 & ns \\
\hline Firmicutes & Eubacteriaceae & Eubacterium & Eubacterium siraeum V10Sc8a & $0.002 \pm 0.001$ & $0.005 \pm 0.000$ & 195.2 & 0.018 & ns \\
\hline Firmicutes & Ruminococcaceae & Ruminococcus & Ruminococcus flavefaciens & $0.009 \pm 0.001$ & $0.004 \pm 0.001$ & -59.1 & 0.006 & ns \\
\hline Firmicutes & Ruminococcaceae & Ruminococcus & Ruminococcus sp. NML 000124 & $0.003 \pm 0.001$ & $0.005 \pm 0.000$ & 82.1 & 0.029 & ns \\
\hline Firmicutes & Ruminococcaceae & Ruminococcus & Ruminococcus faecis & $0.005 \pm 0.000$ & $0.002 \pm 0.001$ & -69.0 & 0.017 & ns \\
\hline Firmicutes & Lachnospiraceae & Blautia & [Ruminococcus] obeum & $0.008 \pm 0.002$ & $0.002 \pm 0.000$ & -76.8 & 0.043 & ns \\
\hline Firmicutes & Lachnospiraceae & Blautia & Blautia sp. Ser5 & $0.006 \pm 0.001$ & $0.002 \pm 0.001$ & -64.6 & 0.033 & ns \\
\hline Firmicutes & Lachnospiraceae & Blautia & Ruminococcus gnavus & $0.004 \pm 0.001$ & $0.001 \pm 0.001$ & -61.0 & 0.028 & ns \\
\hline Firmicutes & Lachnospiraceae & Blautia & [Ruminococcus] torques & $0.001 \pm 0.000$ & $0.001 \pm 0.000$ & 74.9 & 0.044 & ns \\
\hline Firmicutes & Lachnospiraceae & Marvinbryantia & Marvinbryantia formatexigens & $0.008 \pm 0.003$ & $0.001 \pm 0.000$ & -91.7 & 0.049 & ns \\
\hline Firmicutes & Lactobacillaceae & Lactobacillus & Lactobacillus reuteri & $0.001 \pm 0.001$ & $0.005 \pm 0.000$ & 236.8 & 0.016 & ns \\
\hline Proteobacteria & Desulfovibrionaceae & Bilophila & Bilophila wadsworthia & $0.005 \pm 0.000$ & $0.025 \pm 0.005$ & 406.7 & 0.012 & ns \\
\hline Proteobacteria & Xanthomonadaceae & Frateuria & Frateuria aurantia & $0.002 \pm 0.001$ & $0.005 \pm 0.000$ & 229.4 & 0.016 & ns \\
\hline Actinobacteria & Coriobacterineae & Asaccharobacter & Asaccharobacter celatus & $0.005 \pm 0.000$ & $0.002 \pm 0.001$ & -67.2 & 0.016 & ns \\
\hline Bacteroidetes & Prevotellaceae & Prevotella & Prevotella brevis & $0.039 \pm 0.009$ & $0.005 \pm 0.000$ & -87.1 & 0.018 & ns \\
\hline Bacteroidetes & Prevotellaceae & Paraprevotella & Paraprevotella xylaniphila & $0.009 \pm 0.001$ & $0.002 \pm 0.001$ & -78.1 & 0.005 & ns \\
\hline Bacteroidetes & Prevotellaceae & Prevotella & Prevotella micans & $0.002 \pm 0.001$ & $0.005 \pm 0.000$ & 157.6 & 0.004 & ns \\
\hline Bacteroidetes & Prevotellaceae & Prevotella & Prevotella marshii & $0.002 \pm 0.001$ & $0.005 \pm 0.000$ & 205.3 & 0.004 & ns \\
\hline Bacteroidetes & Prevotellaceae & Prevotella & Prevotella copri & $0.002 \pm 0.001$ & $0.005 \pm 0.000$ & 133.1 & 0.037 & ns \\
\hline
\end{tabular}


Table 4. Continued

\begin{tabular}{|c|c|c|c|c|c|c|c|c|}
\hline Phyla & Family & Genus & Bacterial species & HFS baseline & $\begin{array}{l}\text { HFS after } \\
\text { treatment }\end{array}$ & Change (\%) & $\begin{array}{l}\text { Unadjusted } \\
p \text { value }\end{array}$ & $\begin{array}{c}\text { Critical } \\
\text { value }\end{array}$ \\
\hline Bacteroidetes & Prevotellaceae & Prevotella & Prevotella sp. DJF B116 & $0.008 \pm 0.001$ & $0.005 \pm 0.000$ & -38.9 & 0.040 & $\mathrm{~ns}$ \\
\hline Bacteroidetes & Prevotellaceae & Prevotella & Prevotella sp. oral taxon 302 & $0.002 \pm 0.001$ & $0.005 \pm 0.000$ & 114.8 & 0.025 & ns \\
\hline Bacteroidetes & Prevotellaceae & Prevotella & Prevotella sp. B31FD & $0.001 \pm 0.001$ & $0.005 \pm 0.000$ & 247.0 & 0.016 & ns \\
\hline Bacteroidetes & Bacteroidaceae & Bacteroides & Bacteroides heparinolyticus & $0.002 \pm 0.001$ & $0.005 \pm 0.000$ & 204.2 & 0.016 & ns \\
\hline Bacteroidetes & Bacteroidaceae & Bacteroides & Bacteroides sp. S- 18 & $0.002 \pm 0.001$ & $0.040 \pm 0.012$ & 1526.4 & 0.035 & $\mathrm{~ns}$ \\
\hline Bacteroidetes & Bacteroidaceae & Bacteroides & Bacteroides sp. dnLKV7 & $0.003 \pm 0.001$ & $0.013 \pm 0.004$ & 427.8 & 0.043 & $\mathrm{~ns}$ \\
\hline Bacteroidetes & Bacteroidaceae & Bacteroides & Bacteroides sp. Human4 & $0.005 \pm 0.000$ & $0.001 \pm 0.001$ & -71.0 & 0.017 & ns \\
\hline Bacteroidetes & Bacteroidaceae & Bacteroides & Bacteroides dorei & $0.005 \pm 0.000$ & $0.003 \pm 0.001$ & -48.5 & 0.044 & ns \\
\hline Bacteroidetes & Porphyromonadaceae & Barnesiella & Barnesiella intestinihominis & $0.082 \pm 0.012$ & $0.006 \pm 0.003$ & -93.0 & 0.002 & $\mathrm{~ns}$ \\
\hline Bacteroidetes & Porphyromonadaceae & Barnesiella & Barnesiella viscericola & $0.019 \pm 0.004$ & $0.003 \pm 0.001$ & -84.9 & 0.015 & ns \\
\hline Bacteroidetes & Porphyromonadaceae & Porphyromonas & Porphyromonas endodontalis & $0.001 \pm 0.000$ & $0.004 \pm 0.001$ & 244.5 & 0.043 & ns \\
\hline Bacteroidetes & Porphyromonadaceae & Barnesiella & Barnesiella sp. NSB1 & $0.029 \pm 0.006$ & $0.005 \pm 0.003$ & -83.3 & 0.006 & $\mathrm{~ns}$ \\
\hline Bacteroidetes & Flavobacteriaceae & Maribacter & Maribacter sp. ZS1-8 & $0.002 \pm 0.001$ & $0.005 \pm 0.000$ & 189.0 & 0.017 & $\mathrm{~ns}$ \\
\hline Tenericutes & Acholeplasmataceae & Phytoplasma & 16SrIII (X-disease group) & $0.001 \pm 0.001$ & $0.005 \pm 0.000$ & 265.7 & 0.016 & ns \\
\hline Tenericutes & Acholeplasmataceae & Phytoplasma & $\begin{array}{l}\text { 'Spartium junceum' witches'-broom } \\
\text { phytoplasma }\end{array}$ & $0.002 \pm 0.001$ & $0.005 \pm 0.000$ & 206.9 & 0.018 & ns \\
\hline
\end{tabular}

Taxonomic breakdown of Wistar rats intestinal bacterial V4-V6 regions obtained by pyrosequencing before and after HFS diet treatment. All results are expressed as the taxa frequencies mean \pm SEM and the average of bacterial relative abundance change (\%) between final and baseline. Statistical analyses were performed using Paired- Samples T-test. Variables presenting probabilities of $\mathrm{p} \quad 0.05$ were applied for the Benjamini-Hochberg multiple testing corrections. Unadjusted $p$ values lower than the calculated $p$ critical values were considered statistically significant $(*)$. HFS, high-fat sucrose; SEM, standard error of the mean. 


\section{SCFA profiles}

Follow-up GC-MS analysis of faecal contents showed significantly lower $(p<0.05)$ concentrations of acetate $(1.68 \pm 0.39 \mathrm{mg} / \mathrm{g})$ and propionate $(0.41 \pm 0.11 \mathrm{mg} / \mathrm{g})$ at the end of the study compared to the baseline $(6.40 \pm 0.54 ; 1.27 \pm 0.10 \mathrm{mg} / \mathrm{g}$, respectively) while final butyrate levels $(0.38 \pm 0.11 \mathrm{mg} / \mathrm{g})$ present a trend $(p=0.083)$ to be reduced in comparison to the contents measured in faeces at the beginning of the study $(9.46 \pm$ $3.95 \mathrm{mg} / \mathrm{g})$. Moreover, faecal matter collected in $24 \mathrm{~h}$ was significantly $(p=0.042)$ reduced at the end of the treatment $(1.8 \pm 0.6 \mathrm{~g})$ compared to baseline $(7.0 \pm 0.5 \mathrm{~g})$. Thus, absolute amounts of acetate $(44.81 \pm 3.80 \mathrm{mg})$ and propionate $(8.89 \pm 0.71 \mathrm{mg})$ before treatment were significantly higher $(p<0.001)$ and decreased after the HFS diet (acetate, $3.02 \pm 0.70 \mathrm{mg}$; propionate, $0.74 \pm 0.20 \mathrm{mg}$ ), whereas initial total butyrate quantities $(66.21 \pm 27.64 \mathrm{mg})$ showed a trend $(p=0.077)$ to be reduced $(0.69 \pm 0.19 \mathrm{mg})$ after the HFS diet treatment (Figure 6a and 6b).

\section{Discussion}

Gut microbiota composition is usually altered in metabolic diseases (Fukuda and Ohno, 2013). Also, dietary patterns rich in fat and carbohydrates have been reported to modify the bacterial community at high phylogenetic levels (de La Serre et al., 2010; Parks et al., 2013). Furthermore, the bacterial structure modifications as a result of small variations in dietary macronutrients have been characterized (Patrone et al., 2012). In this context, our study aimed to demonstrate the gut microbiota susceptibility to modifications in diet-induced overweight rats at different phylogenetic levels. Moreover, the current research sought to further demonstrate how a Westernized diet might importantly affect the gut microbiota composition, by the implementation of an experimental model fed a diet rich in fat and sucrose that resembles and simulates the overweight and obesity associated metabolic alterations in humans. On the other hand, these results provide information about the role of the diet in gut microbial population of a unique group of animals that has been analysed at two different time points (baseline and at the end of the HFS diet treatment), thereby avoiding inter-individual differences.

16S rRNA sequence-based analytical methods have unveiled that Gram positive Firmicutes and Gram negative Bacteroidetes constitute over 90\% of the known phylogenetic categories and are the dominant divisions in the distal gastrointestinal tract from human volunteers (Qin et al., 2010). This trial confirms that the consumption of a HFS diet during 6 weeks causes a restructuring of gut microbial community in rats, supporting data from other authors (Parks et al., 2013) that assure the differential characterization of gut microbiota between standard diet-fed lean rats and HFS dietinduced obese rats. In this sense, our study demonstrates that the HFS diet treatment causes a significant increase of bacteria belonging to the phylum Firmicutes and Proteobacteria, accompanied with a division-wide suppression of Bacteroidetes. These data agree with the obesity-associated compositional pattern reported by Gordon and colleagues (Ley et al., 2005) for obese humans and obese (ob/ob) mice, finding the increase in the ratio Firmicutes to Bacteroidetes to be higher at the end of the HFS intervention. The overweight state has been previously characterized by reduced proportions of the phylum Bacteroidetes and equivalent enrichments in Firmicutes (increased Firmicutes-to-Bacteroidetes ratio) (Ley et al., 2005; Ley et al., 2006; Murphy et al., 2010). However, this assumption is under debate as some groups were not able to detect differences between obese and non-obese subjects for the phylum 
Bacteroidetes (Duncan et al., 2008), other researchers demonstrated the opposite results by reporting that obese subjects harboured more bacteria from this phylum than normalweight individuals (Zhang et al., 2009) and other investigators have even found lower Firmicutes to Bacteroidetes ratio (Schwiertz et al., 2010). On the other hand, in this study a significant enrichment in Proteobacteria was also found, which is consistent with the results published by Hildebrandt et al. (Hildebrandt et al., 2009). This group elegantly demonstrated that the effect of the diet dominated over the obese state on gut microbiome composition. The authors took advantage of the use of RELM $\beta$ KO mice, which remained lean when fed a high-fat diet, and compared the results obtained with high-fat diet fed wild type mice. Our findings are similar to those reported by this group, as the authors demonstrated an increase in the abundance of the Proteobacteria phylum.

Not only the whole Firmicutes phylum, but a predominant class (the Mollicutes) within this group has been associated to western-diet related gut microbiota (Turnbaugh et al., 2008). Our data agree with previous metagenomic analyses performed in diet-induced obese mice showing a bloom in the Erysipelotrichi class within the Firmicutes phylum (Fleissner et al., 2010; Turnbaugh et al., 2008; Turnbaugh et al., 2009). In this context, it has been hypothesized that this group of bacteria could has the capacity to take up oligo- and monosaccharides from Western diets, such as glucose, fructose and sucrose, in order to produce ready to absorb short-chain fatty acids by their metabolism (Conterno et al., 2011). Moreover, these data should also consider the relevance of the increased body weight and possible metabolic changes related to the acquired overweight as a possible explanation for the increased Erysipelotrichi abundance. In this sense, abundance of the taxa Erysipelotrichiciae, together with the levels of Coriobacteriaceae and Gammaproteobacteria, has also been associated to alterations in lipid metabolism in the context of obesity, metabolic syndrome, and hypercholesterolemia (Martinez et al., 2013; Spencer et al., 2011). However, it could not be concluded that the enrichment in Erysipelotrichiciae detected in our study, is related to the host lipid and cholesterol phenotypes as we do not have biochemical data from these variables at the beginning of the study. Anyway, our group has previously reported a marked increase in body weight, subcutaneous and visceral fat depots due to the HFS diet in this experimental model that is accompanied by singular metabolic impairments (Boque et al., 2013). Therefore, it is likely that the metabolic derangement caused by the increased intake of fat and sugars given by the HFS diet could have produced a direct effect on this specific taxa. Indeed, the fact that the gut microbiota composition could be independent of the host phenotype, with the diet being the major contributory factor for its altered composition, has been previously reported in weightreduced mice (Ravussin et al., 2012), and also in lean/ obese mice phenotypes (Hildebrandt et al., 2009). In this sense, an enhancement in Delta-Proteobacteria class and the order Desulfovibrio as a consequence of the high-fat diet was demonstrated by Hildebrandt et al. (Hildebrandt et al., 2009). In agreement with these findings, an enlargement in the Desulforvibrionaceae family after the HFS diet treatment was also detected in our study after the HFS diet treatment. It should be highlighted that the family Desulfovibrionaceae, in spite of not being so well known, has been described as a sulphate-reducing, endotoxin-producing bacteria and has been positively associated with obesity and/or type 2 diabetes (Hildebrandt et al., 2009; Zhang et al., 2010). Furthermore, significant reductions observed in the Bacteroidia class and the Prevotellaceae family in our study, are in accordance with the HFS diet-induced decreases found by Hildebrandt et al. (Hildebrandt et al., 2009) in the families of Bacteroidaceae, Prevotellaceae and Rikenellaceae. Moreover, our data evidenced a 
lower Prevotellaceae/Bacteroidaceae ratio at the end of the intervention period. Interestingly, this ratio has been previously reported to be increased after a weight loss intervention (Nadal et al., 2009; Santacruz et al., 2009).

Nevertheless, there is a key bacterial group that has been found to differ in our study when compared to other studies (de La Serre et al., 2010; Hildebrandt et al., 2009). Our results showed a significant decrease in Clostridia after the HFS diet treatment, while Hildebrandt et al. (Hildebrandt et al., 2009) and other authors (de La Serre et al., 2010) have reported an enhancement of this bacterial group in high-fat diet-fed mice and rats. We speculate that the disparity found regarding Clostridia class, might originate from differences in the carbohydrate contents of the diets. As mentioned in material and methods sections, our rats were fed a standard diet at the beginning of the intervention (low in fat and simple sugars, high in fiber) and then switched to a high-fat, highsucrose diet. This variation in the type and content of carbohydrates could cause changes in specific bacterial groups, such as the Clostridia class, which differs from the alterations produced by a purified high-fat diet. In fact, it has been demonstrated that Clostridium, which is an obligate anaerobe genus, is susceptible to changes associated to the digestible carbohydrate content of the diet, (i.e. corn starch), their counts being increased with high starch diets (Cresci et al., 1999). This finding may assume a possible direct impact of the type and quantity of carbohydrates on gut microbiota composition. Supporting this hypothesis, Fleissner et al. (Fleissner et al., 2010) studied the influence of a standard (SD), a high-fat (HF) and a commercial Western diet (WD) on faecal microbiota composition of conventional mice. Our data are consistent with the reductions of bacterial families reported by them in the HF and WD-fed animals, including Bacillaceae, Lachnospiraceae, Lactobacillaceae, Porphyromonadaceae, Rikenellaceae, Prevotellaceae and Bacteroidaceae. Interestingly, the gut microbiota of $\mathrm{HF}$ and WD-fed mice was enriched in the Erysipelotrichiciae family as previously mentioned, but the Clostridiaceae family suffered a decrease when the mice were fed the WD.

The consequences of the fermentation of different types of carbohydrates have been extensively analysed elsewhere (den Besten et al., 2013; Louis et al., 2007). Non-digestible carbohydrates reach intact the cecum and are key constituents to modify gut microbiota composition and hence, the type and amount of SCFA produced (Campbell et al., 1997). This study revealed a significant reduction in the total amount of acetate and propionate, but only a tendency to decrease was found for butyrate. The latter might be due to the high- variability given within animals in the levels of this metabolite at baseline. Moreover, it should also be taken into consideration that as a consequence of the lower total amount of faecal matter collected at the end of the study compared to baseline a considerable reduction in the absolute amounts of SCFA was found. Indeed, these differences in faecal matter might be partly attributed to the constipation that the HFS diet could produce in animals resulting from a lower fiber and resistant starch content. Nonetheless, these results contradict data from previously reported experiments (Ley et al., 2005; Ley et al., 2006; Turnbaugh et al., 2006). Briefly, Turnbaugh et al. (Turnbaugh et al., 2006) characterized the distal gut microbiota differences in $o b / o b$ mice and their lean littermates $(o b /+,+/+)$ removing confounding factors such as, environmental, dietary and genotype factors. The authors hypothesized that the $\mathrm{C} 57 \mathrm{BL} / 6 \mathrm{~J} o b / o b$ genotype had a greater capacity to energy harvest from the diet. To test this hypothesis, caecal microbiota from C57BL/6J lean and obese donors was transplanted to germ-free C5BL/6J recipients. Such findings confirmed that the donor microbiota was transmissible. In addition, it was demonstrated that those 
animals who received the obese microbiota, showed a significantly higher percentage increase in body fat and a greater production of SCFA in the caeca of conventionally raised C57BL/6J mice. Altogether, this study concluded that the obese phenotype is transmissible and present an enlarged capacity to extract energy from the diet. Concerning our outcomes, the possible main reason for the observed differences in the detected SCFA, might be behind the experimental model used. The above mentioned studies were performed in genetically obese phenotypes, homozygous for a mutation in the leptin gene $\left(\mathrm{Lep}^{\mathrm{ob}}\right)$, which produces obesity regardless of food intake, whereas our model, depends on the amount and type of fat and carbohydrates consumed to develop the disease, which in turn, may affect microbial composition and their primary fermentation products. Nevertheless, it is important to remark that these measurements in faeces are useful when talking about changes in excretion, but are imprecise if production of these molecules are to be discussed, since their content in faeces depends on the absorption by the host and is influenced by rates of transit (Topping and Clifton, 2001).

Approaches based on 16S rRNA (16S rDNA) analyses have enabled to detect taxonomic differences in microbial communities at phylum level, but also have demonstrated to be valuable tools to identify genus- and species-specific alterations (Lamendella et al., 2012). Moreover, clustering of bacterial families based on PCA, showed a clear representation of differentiated lineages of bacteria between samples collected before the intervention period when rats were fed a control-diet during the adaptation period, and HFS diet-fed overweight rats. On the other hand, the evaluation of gut microbiota composition in faecal samples from lean and diet-induced overweight rats, demonstrated that there are specific bacterial species that present considerable percentages of change at the end of the treatment when compared to the baseline. One of these species is Mitsuokella jalaludinii, significantly depleted after the HFS treatment. Mitsuokella jalaludinii, has been recently isolated from the rumen of cattle (Lan et al., 2002) and is a member of the Veillonellaceae family that has been previously demonstrated to be reduced in HFD-fed rats (Yin et al., 2013). In our experiment, Veillonellaceae did not statistically differ between groups but, as observed in the PCA, it was represented in a greater extent in HFS diet-fed rats. On the other hand, Bilophila wadsworthia from Desulfovibrionaceae family was increased after the HFS treatment in our rats. Bilophila wadsworthia is defined as a sulphate-reducing, bile resistant bacteria, difficult to detect in healthy individuals (Devkota et al., 2012) while it has been reported to increase under inflammatory conditions (Devkota et al., 2012). Moreover, the growth of this bacterium has been related to the suppression of symbiotic, commensal microbes, which might allow pathobionts and pathogens to establish in the intestine (Devkota et al., 2012). The relative abundance of other bacterial species, such as Prevotella micans, Prevotella marshii, Prevotella sp. oral taxon 302 and Prevotella sp. B31 FD and especially Prevotella nanceiensis were found to be increased after the intervention, even if the relative levels of the Prevotellaceae family were found to be reduced. Members of this family are classified as acetate and $\mathrm{H}_{2}$-producers (Marounek and Dušková, 1999) and, contrarily to what we observed in rats, have been found to be enriched in obese human subjects (Zhang et al., 2009). Nevertheless, dietary patterns such as high fiber intake have been reported to impact in the abundance of this group (De Filippo et al., 2010). Therefore, taking into account that Prevotella nanceiensis is able to ferment saccharides such as sucrose, mannose and raffinose (Alauzet et al., 2007), the increases detected in this bacterial species and related members, could be attributed to dietary macronutrients (carbohydrates) modifications. On the other hand, PCA showed that Clostridiaceae family was greatly 
present in faecal samples from control diet-fed lean rats. However, a significant expansion in the levels of Clostridium sp. FCB90-3, Clostridium sp. BNL1100, as well as a slight increase in Clostridium algidixylanolyticum and Clostridium sp. C9 members within this family was detected at the end of the intervention in our study. In contrast, our results revealed that, the HFS diet negatively affected Clostridium species belonging to Clostridia cluster XIVa (Clostridium hathewayi, Clostridium aerotolerans, Clostridium xylanovorans and [Ruminococcus] obeum), although the reduction was statistically significant only for Clostridium fusiformis. Bacterial species from this group have been demonstrated to be negatively affected in obesity (Cani and Delzenne, 2009). Indeed, they are major producers of SCFA (that decreased with the HFS diet), especially butyrate, which has been shown to play a role in the protection against harmful inflammatory responses (Donohoe et al., 2011). In addition, the decreases found in the levels of Clostridium clariflavum, a cellulose-digesting bacteria classified within the Clostridium cluster III, denotes the potential influence of the diet or specific nutrients on gut microbial community (Shiratori et al., 2009). Other members from the Firmicutes phylum, Eubacterium ventriosum and Eubacterium cylindroides, were considerably increased after the experimental period in faeces from the HFS-induced overweight rats. Interestingly, Eubacterium ventriosum has been positively correlated to body mass index (Tims et al., 2013) while Eubacterium cylindroides, is a member of the Erysipelotrichiciae family, and probably the major contributor to a high abundance in our study. Moreover, this bacterium has been reported to be up regulated in high-fat diet induced obesity and in faecal microbiota of obese humans (Ley et al., 2005; Turnbaugh et al., 2008). Finally, higher levels of Lactobacillus reuteri at the end of the intervention (Lactobacillaceae family) were found in agreement with previous studies (Million et al., 2012).

Our data indicate that gut microbial ecology, apart from being modified at highest division levels, responds to the diet in a species-dependent manner. Furthermore, it is noteworthy that differential response patterns could be given when phylogenetic analyses are conducted at deeper levels. Herein, we attempted to characterize the gut microbiota modifications resulted from the intake of an obesogenic diet in Wistar rats. It should be emphasized that in this experiment, the same group of animals' was tested at different time points of the study (baseline and after the HFS diet treatment). This way, the problem of inter-individual variability was overcome as genetic differences within animals were avoided, and the identical "inherited" pattern of microbes was ensured. Nevertheless, there are several limitations that should be mentioned. Firstly, we did not have a separate group of rats that were fed a standard diet for the same period of time (6 weeks) that would serve as a control group. Moreover, the sample size used in the study $(n=5)$ was small providing a low statistical power after the correction for multiple comparison. In this regard, the low power of the study could lead to Type II Error or false negatives. Nevertheless, it was enough to detect reported differences, thus it could be mentioned that the weakness associated to the small sample size was that we likely failed to identify more statistical significances in other comparisons performed. Furthermore, we are also concerned that the sample size was small based on the demonstrated genetic variance in rats (Levin, 1990) that could have made some animals prone to obesity and others resistant to obesity when fed the high-energy diet. Secondly, we have only observed the modifications produced by a unique diet, thus the relevance of different components from the diet cannot be separately elucidated. Finally, it is remarkable that this study has been conducted in rats whereas microbiota analyses from most of the cited articles have been performed in mice or in humans. Thus, disparities 
with other studies could be due to factors such as diet, age, degree of obesity, sample size, sample collection and manipulation, techniques and methodology used that could profoundly affect gut microbes identification. Still, this study brings light to the fact that specific species (Mitsuokella jalaludinii, Eubacterium ventriosum, Prevotella nanceiensis, Eubacterium cylindroides and some Clostridium species), detected by a high-throughput technique, are affected by an intervention period with a HFS diet in Wistar rats, as well as the widely reported Firmicutes, Bacteroidetes, Proteobacteria phyla and Erysipelotrichi class.

\section{Acknowledgements}

This study was supported by grants from the Ministerio de Economía y Competitividad (AGL2011-27406-ALI), Instituto de Salud Carlos III (CIBERobn), Government of the Basque Country (IT-572-13) and University of the Basque Country (UPV/EHU) (ELDUNANOTEK UFI11/32). The authors wish to acknowledge Línea Especial about Nutrition, Obesity and Health (University of Navarra LE/97, Spain) for the financial support and the Department of Education, Language policy and Culture from Government of the Basque Country for the predoctoral grant given to Usune Etxeberria.

\section{References}

Alauzet, C., Mory, F., Carlier, J.P., Marchandin, H., Jumas-Bilak, E. and Lozniewski, A., 2007. Prevotella nanceiensis sp. nov., isolated from human clinical samples. Int J Syst Evol Microbiol 57: 2216-2220.

Azagury, D.E. and Lautz, D.B., 2011. Obesity overview: epidemiology, health and financial impact, and guidelines for qualification for surgical therapy. Gastrointest Endosc Clin N Am 21: 189-201.

Boque, N., Campion, J., de la Iglesia, R., de la Garza, A.L., Milagro, F.I., San Roman, B., Banuelos, O. and Martinez, J.A., 2013. Screening of polyphenolic plant extracts for anti-obesity properties in Wistar rats. J Sci Food Agric 93: 1226-1232.

Campbell, J.M., Fahey, G.C., Jr. and Wolf, B.W., 1997. Selected indigestible oligosaccharides affect large bowel mass, cecal and fecal short-chain fatty acids, $\mathrm{pH}$ and microflora in rats. J Nutr 127: 130-136.

Cani, P.D. and Delzenne, N.M., 2009. Interplay between obesity and associated metabolic disorders: new insights into the gut microbiota. Curr Opin Pharmacol 9: 737-743.

Cani, P.D. and Delzenne, N.M., 2011. The gut microbiome as therapeutic target. Pharmacol Ther 130: 202-212.

Cani, P.D., Everard, A. and Duparc, T., 2013. Gut microbiota, enteroendocrine functions and metabolism. Curr Opin Pharmacol 13: 935-940.

Chevreux, B., Pfisterer, T., Drescher, B., Driesel, A.J., Muller, W.E., Wetter, T. and Suhai, S., 2004. Using the miraEST assembler for reliable and automated mRNA transcript assembly and SNP detection in sequenced ESTs. Genome Res 14: 1147-1159.

Conterno, L., Fava, F., Viola, R. and Tuohy, K.M., 2011. Obesity and the gut microbiota: does up-regulating colonic fermentation protect against obesity and metabolic disease? Genes Nutr 6: 241-260.

Cresci, A., Orpianesi, C., Silvi, S., Mastrandrea, V. and Dolara, P., 1999. The effect of sucrose or starch-based diet on short-chain fatty acids and faecal microflora in rats. J Appl Microbiol 86: 245-250.

Daniel, H., Gholami, A.M., Berry, D., Desmarchelier, C., Hahne, H., Loh, G., Mondot, S., Lepage, P., Rothballer, M., Walker, A., Bohm, C., Wenning, M., Wagner, M., Blaut, M., SchmittKopplin, P., Kuster, B., Haller, D. and Clavel, T., 2013. High-fat diet alters gut microbiota physiology in mice. ISME J. 
De Filippo, C., Cavalieri, D., Di Paola, M., Ramazzotti, M., Poullet, J.B., Massart, S., Collini, S., Pieraccini, G. and Lionetti, P., 2010. Impact of diet in shaping gut microbiota revealed by a comparative study in children from Europe and rural Africa. Proc Natl Acad Sci U S A 107: 14691-14696.

de La Serre, C.B., Ellis, C.L., Lee, J., Hartman, A.L., Rutledge, J.C. and Raybould, H.E., 2010. Propensity to high-fat diet-induced obesity in rats is associated with changes in the gut microbiota and gut inflammation. Am J Physiol Gastrointest Liver Physiol 299: G440448.

den Besten, G., van Eunen, K., Groen, A.K., Venema, K., Reijngoud, D.J. and Bakker, B.M., 2013. The role of short-chain fatty acids in the interplay between diet, gut microbiota, and host energy metabolism. J Lipid Res 54: 2325-2340.

Devkota, S., Wang, Y., Musch, M.W., Leone, V., Fehlner-Peach, H., Nadimpalli, A., Antonopoulos, D.A., Jabri, B. and Chang, E.B., 2012. Dietary-fat-induced taurocholic acid promotes pathobiont expansion and colitis in Il10-/- mice. Nature 487: 104-108.

Donohoe, D.R., Garge, N., Zhang, X., Sun, W., O'Connell, T.M., Bunger, M.K. and Bultman, S.J., 2011. The microbiome and butyrate regulate energy metabolism and autophagy in the mammalian colon. Cell Metab 13: 517-526.

Duca, F.A., Sakar, Y. and Covasa, M., 2013. The modulatory role of high fat feeding on gastrointestinal signals in obesity. J Nutr Biochem 24: 1663-1677.

Duncan, S.H., Lobley, G.E., Holtrop, G., Ince, J., Johnstone, A.M., Louis, P. and Flint, H.J., 2008. Human colonic microbiota associated with diet, obesity and weight loss. Int J Obes (Lond) 32: 1720-1724.

Evans, J.M., Morris, L.S. and Marchesi, J.R., 2013. The gut microbiome: the role of a virtual organ in the endocrinology of the host. J Endocrinol 218: R37-47.

Fleissner, C.K., Huebel, N., Abd El-Bary, M.M., Loh, G., Klaus, S. and Blaut, M., 2010. Absence of intestinal microbiota does not protect mice from diet-induced obesity. $\mathrm{Br} J$ Nutr 104: 919-929.

Flint, H.J., Bayer, E.A., Rincon, M.T., Lamed, R. and White, B.A., 2008. Polysaccharide utilization by gut bacteria: potential for new insights from genomic analysis. Nat Rev Microbiol 6: 121-131.

Fukuda, S. and Ohno, H., 2013. Gut microbiome and metabolic diseases. Semin Immunopathol. Doi: $10.1007 / \mathrm{s} 00281-013-0399-z$

Hildebrandt, M.A., Hoffmann, C., Sherrill-Mix, S.A., Keilbaugh, S.A., Hamady, M., Chen, Y.Y., Knight, R., Ahima, R.S., Bushman, F. and Wu, G.D., 2009. High-fat diet determines the composition of the murine gut microbiome independently of obesity. Gastroenterology 137: 1716-1724 e1711-1712.

Huson, D.H., Mitra, S., Ruscheweyh, H.J., Weber, N. and Schuster, S.C., 2011. Integrative analysis of environmental sequences using MEGAN4. Genome Res 21: 1552-1560.

Lamendella, R., VerBerkmoes, N. and Jansson, J.K., 2012. 'Omics' of the mammalian gut--new insights into function. Curr Opin Biotechnol 23: 491-500.

Lan, G.Q., Abdullah, N., Jalaludin, S. and Ho, Y.W., 2002. Culture conditions influencing phytase production of Mitsuokella jalaludinii, a new bacterial species from the rumen of cattle. J Appl Microbiol 93: 668-674.

Layden, B.T., Angueira, A.R., Brodsky, M., Durai, V. and Lowe, W.L., Jr., 2013. Short chain fatty acids and their receptors: new metabolic targets. Transl Res 161: 131-140.

Levin, B.E., 1990. Obesity-prone and -resistant rats differ in their brain [3H]paraminoclonidine binding. Brain Res 512: 54-59.

Ley, R.E., 2010. Obesity and the human microbiome. Curr Opin Gastroenterol 26: 5-11.

Ley, R.E., Backhed, F., Turnbaugh, P., Lozupone, C.A., Knight, R.D. and Gordon, J.I., 2005. Obesity alters gut microbial ecology. Proc Natl Acad Sci U S A 102: 11070-11075.

Ley, R.E., Turnbaugh, P.J., Klein, S. and Gordon, J.I., 2006. Microbial ecology: human gut microbes associated with obesity. Nature 444: 1022-1023. 
Lomba, A., Milagro, F.I., Garcia-Diaz, D.F., Marti, A., Campion, J. and Martinez, J.A., 2010. Obesity induced by a pair-fed high fat sucrose diet: methylation and expression pattern of genes related to energy homeostasis. Lipids Health Dis 9: 60.

Louis, P., Scott, K.P., Duncan, S.H. and Flint, H.J., 2007. Understanding the effects of diet on bacterial metabolism in the large intestine. J Appl Microbiol 102: 1197-1208.

Macfarlane, S. and Macfarlane, G.T., 2003. Regulation of short-chain fatty acid production. Proc Nutr Soc 62: 67-72.

Marounek, M. and Dušková, D., 1999. Metabolism of pectin in rumen bacteria Butyrivibrio fibrisolvens and Prevotella ruminicola. Letters in Applied Microbiology 29: 429-433.

Martinez, I., Perdicaro, D.J., Brown, A.W., Hammons, S., Carden, T.J., Carr, T.P., Eskridge, K.M. and Walter, J., 2013. Diet-induced alterations of host cholesterol metabolism are likely to affect the gut microbiota composition in hamsters. Appl Environ Microbiol 79: 516524.

Martinez, J.A., Cordero, P., Campion, J. and Milagro, F.I., 2012. Interplay of early-life nutritional programming on obesity, inflammation and epigenetic outcomes. Proc Nutr Soc 71: 276-283.

Milagro, F.I., Campion, J., Cordero, P., Goyenechea, E., Gomez-Uriz, A.M., Abete, I., Zulet, M.A. and Martinez, J.A., 2011. A dual epigenomic approach for the search of obesity biomarkers: DNA methylation in relation to diet-induced weight loss. FASEB J 25: 13781389.

Million, M., Lagier, J.C., Yahav, D. and Paul, M., 2013. Gut bacterial microbiota and obesity. Clin Microbiol Infect 19: 305-313.

Million, M., Maraninchi, M., Henry, M., Armougom, F., Richet, H., Carrieri, P., Valero, R., Raccah, D., Vialettes, B. and Raoult, D., 2012. Obesity-associated gut microbiota is enriched in Lactobacillus reuteri and depleted in Bifidobacterium animalis and Methanobrevibacter smithii. Int J Obes (Lond) 36: 817-825.

Murphy, E.F., Cotter, P.D., Healy, S., Marques, T.M., O'Sullivan, O., Fouhy, F., Clarke, S.F., O'Toole, P.W., Quigley, E.M., Stanton, C., Ross, P.R., O'Doherty, R.M. and Shanahan, F., 2010. Composition and energy harvesting capacity of the gut microbiota: relationship to diet, obesity and time in mouse models. Gut 59: 1635-1642.

Nadal, I., Santacruz, A., Marcos, A., Warnberg, J., Garagorri, J.M., Moreno, L.A., MartinMatillas, M., Campoy, C., Marti, A., Moleres, A., Delgado, M., Veiga, O.L., GarciaFuentes, M., Redondo, C.G. and Sanz, Y., 2009. Shifts in clostridia, bacteroides and immunoglobulin-coating fecal bacteria associated with weight loss in obese adolescents. Int J Obes (Lond) 33: 758-767.

Parks, B.W., Nam, E., Org, E., Kostem, E., Norheim, F., Hui, S.T., Pan, C., Civelek, M., Rau, C.D., Bennett, B.J., Mehrabian, M., Ursell, L.K., He, A., Castellani, L.W., Zinker, B., Kirby, M., Drake, T.A., Drevon, C.A., Knight, R., Gargalovic, P., Kirchgessner, T., Eskin, E. and Lusis, A.J., 2013. Genetic control of obesity and gut microbiota composition in response to high-fat, high-sucrose diet in mice. Cell Metab 17: 141-152.

Patrone, V., Ferrari, S., Lizier, M., Lucchini, F., Minuti, A., Tondelli, B., Trevisi, E., Rossi, F. and Callegari, M.L., 2012. Short-term modifications in the distal gut microbiota of weaning mice induced by a high-fat diet. Microbiology 158: 983-992.

Qin, J., Li, R., Raes, J., Arumugam, M., Burgdorf, K.S., Manichanh, C., Nielsen, T., Pons, N., Levenez, F., Yamada, T., Mende, D.R., Li, J., Xu, J., Li, S., Li, D., Cao, J., Wang, B., Liang, H., Zheng, H., Xie, Y., Tap, J., Lepage, P., Bertalan, M., Batto, J.M., Hansen, T., Le Paslier, D., Linneberg, A., Nielsen, H.B., Pelletier, E., Renault, P., Sicheritz-Ponten, T., Turner, K., Zhu, H., Yu, C., Jian, M., Zhou, Y., Li, Y., Zhang, X., Qin, N., Yang, H., Wang, J., Brunak, S., Dore, J., Guarner, F., Kristiansen, K., Pedersen, O., Parkhill, J., Weissenbach, J., Bork, P. and Ehrlich, S.D., 2010. A human gut microbial gene catalogue established by metagenomic sequencing. Nature 464: 59-65. 
Ravussin, Y., Koren, O., Spor, A., LeDuc, C., Gutman, R., Stombaugh, J., Knight, R., Ley, R.E. and Leibel, R.L., 2012. Responses of gut microbiota to diet composition and weight loss in lean and obese mice. Obesity (Silver Spring) 20: 738-747.

Santacruz, A., Marcos, A., Warnberg, J., Marti, A., Martin-Matillas, M., Campoy, C., Moreno, L.A., Veiga, O., Redondo-Figuero, C., Garagorri, J.M., Azcona, C., Delgado, M., GarciaFuentes, M., Collado, M.C. and Sanz, Y., 2009. Interplay between weight loss and gut microbiota composition in overweight adolescents. Obesity (Silver Spring) 17: 19061915.

Schwiertz, A., Taras, D., Schafer, K., Beijer, S., Bos, N.A., Donus, C. and Hardt, P.D., 2010. Microbiota and SCFA in lean and overweight healthy subjects. Obesity (Silver Spring) 18: 190-195.

Shiratori, H., Sasaya, K., Ohiwa, H., Ikeno, H., Ayame, S., Kataoka, N., Miya, A., Beppu, T. and Ueda, K., 2009. Clostridium clariflavum sp. nov. and Clostridium caenicola sp. nov., moderately thermophilic, cellulose-/cellobiose-digesting bacteria isolated from methanogenic sludge. Int J Syst Evol Microbiol 59: 1764-1770.

Spencer, M.D., Hamp, T.J., Reid, R.W., Fischer, L.M., Zeisel, S.H. and Fodor, A.A., 2011. Association between composition of the human gastrointestinal microbiome and development of fatty liver with choline deficiency. Gastroenterology 140: 976-986.

Tagliabue, A. and Elli, M., 2013. The role of gut microbiota in human obesity: recent findings and future perspectives. Nutr Metab Cardiovasc Dis 23: 160-168.

Tims, S., Derom, C., Jonkers, D.M., Vlietinck, R., Saris, W.H., Kleerebezem, M., de Vos, W.M. and Zoetendal, E.G., 2013. Microbiota conservation and BMI signatures in adult monozygotic twins. ISME J 7: 707-717.

Topping, D.L. and Clifton, P.M., 2001. Short-chain fatty acids and human colonic function: roles of resistant starch and nonstarch polysaccharides. Physiol Rev 81: 1031-1064.

Tremaroli, V. and Backhed, F., 2012. Functional interactions between the gut microbiota and host metabolism. Nature 489: 242-249.

Turnbaugh, P.J., Backhed, F., Fulton, L. and Gordon, J.I., 2008. Diet-induced obesity is linked to marked but reversible alterations in the mouse distal gut microbiome. Cell Host Microbe 3: 213-223.

Turnbaugh, P.J., Ley, R.E., Mahowald, M.A., Magrini, V., Mardis, E.R. and Gordon, J.I., 2006. An obesity-associated gut microbiome with increased capacity for energy harvest. Nature 444: 1027-1031.

Turnbaugh, P.J., Ridaura, V.K., Faith, J.J., Rey, F.E., Knight, R. and Gordon, J.I., 2009. The effect of diet on the human gut microbiome: a metagenomic analysis in humanized gnotobiotic mice. Sci Transl Med 1: 6ra14.

Wu, G.D., Chen, J., Hoffmann, C., Bittinger, K., Chen, Y.Y., Keilbaugh, S.A., Bewtra, M., Knights, D., Walters, W.A., Knight, R., Sinha, R., Gilroy, E., Gupta, K., Baldassano, R., Nessel, L., Li, H., Bushman, F.D. and Lewis, J.D., 2011. Linking long-term dietary patterns with gut microbial enterotypes. Science 334: 105-108.

Y Lee, C., 2013. The Effect of High-Fat Diet-Induced Pathophysiological Changes in the Gut on Obesity: What Should be the Ideal Treatment[quest]. Clin Trans Gastroenterol 4: e39.

Yin, X., Peng, J., Zhao, L., Yu, Y., Zhang, X., Liu, P., Feng, Q., Hu, Y. and Pang, X., 2013. Structural changes of gut microbiota in a rat non-alcoholic fatty liver disease model treated with a Chinese herbal formula. Syst Appl Microbiol 36: 188-196.

Zhang, C., Zhang, M., Wang, S., Han, R., Cao, Y., Hua, W., Mao, Y., Zhang, X., Pang, X., Wei, C., Zhao, G., Chen, Y. and Zhao, L., 2010. Interactions between gut microbiota, host genetics and diet relevant to development of metabolic syndromes in mice. ISME J 4: 232-241.

Zhang, H., DiBaise, J.K., Zuccolo, A., Kudrna, D., Braidotti, M., Yu, Y., Parameswaran, P., Crowell, M.D., Wing, R., Rittmann, B.E. and Krajmalnik-Brown, R., 2009. Human gut microbiota in obesity and after gastric bypass. Proc Natl Acad Sci U S A 106: 2365-2370. 